\title{
Improving Loadpull Measurement Time by Intelligent Measurement Interpolation and Surface Modeling Techniques
}

\author{
Paul Hart, John Wood, Basim Noori, Peter Aaen \\ Freescale Semiconductor, Inc., RF Division, 2100 E Elliot Rd, Tempe, AZ 85284 \\ Paul.Hart@freescale.com Tel: (480) 413-3107
}

\begin{abstract}
In this paper we show how a thin-plate spline approximation can be used to generate a model of the measured response surface of a load-pull measurement over a much-reduced number of impedance points with no significant loss of accuracy. Further, interpolation between these model surfaces is possible, generating accurate drive-up characteristics. This has enabled accurate load-pull characterizations to be made in a fraction of the usual time.
\end{abstract}

\section{INTRODUCTION}

Over the past two decades active and passive loadpull measurement techniques have become an invaluable method of high frequency power device characterization. Load-pull measurement techniques can be applied to analyze device behavior as a function of fundamental source and load impedance, DC bias, RF drive level, frequency, test signal type, harmonic termination or any combination thereof. The advantages of such a broad characterization are obvious, however, performing a full device characterization across multiple input parameters can be a very time consuming process involving hundreds or thousands of individual measurements.

In this paper we show how surface modeling and spline interpolation techniques can be applied to greatly reduce the number of measurements and thus the test time required to characterize completely an individual device. Thin-plate splines can be used to model source or load impedance surfaces of constant power, efficiency, linearity or any other parameter that can be measured. We also show how interpolation between several of these surfaces can be used to allow the device performance to be viewed as a function of an additional variable such as power level, bias or frequency. In this way, device performance can be very accurately estimated over a wide range of input variables but using a much-reduced measured data-set.

\section{BACKGROUND \& METHODOLOGY}

Characterization of a device requires the measurement of the device responses to inputs at many discrete data points. In a load-pull characterization the data points are the real and imaginary parts of the load (or source) impedance. To improve the accuracy of the characterization, we may choose to make measurements at very closely-spaced impedance points. This is an expensive exercise in terms of time and resource usage.
Instead, we would prefer to make fewer measurements and then be able to approximate the responses at impedances in between the measured points. There are a variety of global multivariate approximators that can be applied to such a problem, including artificial neural networks, radial basis function networks, etc. Polynomials are often the first-choice of function when smooth data is to be approximated. This is a good choice for local approximation, but over a large space the polynomial may need to be of high degree. Whereas this can result in a polynomial function that is correct at the measured data points, in between these points there may be significant error [1]. Such behavior has resulted in the development of the sophisticated global approximators such as those listed above. The simpler answer to this problem is to use local low-order polynomial approximations that are stitched together to create a smooth curve. In this way, the data in between the measured points can be approximated accurately: such piecewise polynomial interpolating functions are known as splines [2].

For approximating measured load-pull data with a spline we require a two-dimensional approximant. Multivariate splines can be obtained from simple splines by tensor product. This can be a complex procedure, and often care needs to be exercised in the decision of which dimension is to be approximated first, though for load-pull data this is not a significant issue. A simpler bivariate spline construction is provided in the MATLAB $^{\mathrm{TM}}$ Spline Toolbox [3]: the thin-plate smoothing spline. This is in fact a Radial Basis Function approximation:

$$
f(x)=\sum_{j=1}^{n-3} a_{j} \Psi\left(x-c_{j}\right)+\text { linear terms }
$$

in which the $a_{j}$ are coefficients, the $c_{j}$ are centers in the data space, and $\Psi(x)=\left|x^{2}\right| \log \left(x^{2}\right)$ is the radiallysymmetric function. In this expression the summation runs to (n-3), with the remaining terms being accommodated by the linear terms in the expression. The thin-plate spline minimizes the following expression over the function $f$ :

$$
\mathrm{p} \sum_{i=1}^{n-3}\left|y_{i}-f c_{i}^{2}\right|+(1-\mathrm{p}) \int\left(\left|D_{1} D_{1} f\right|^{2}+2\left|D_{1} D_{2} f\right|^{2}+\left|D_{2} D_{2} f\right|^{2}\right)
$$


The $y_{i}$ are the measured response values at the (load-pull impedance) data points $c_{i}$; $\mathrm{p}$ is a smoothing parameter; and $D_{j} f$ denotes the partial derivative of the function $f$ with respect to the impedance plane, where $j=1$ is the real axis and $j=2$ is the imaginary axis of the load-pull impedance: in other words the slope of the spline function in the directions of real and imaginary impedance components.

The measured response data at discrete data points is approximated with a thin plate spline, using the MATLAB ${ }^{\mathrm{TM}}$ Spline Toolbox function. The resulting surface is very smooth, as anticipated: this is shown in Fig.1, and this can be compared with the result from the MATLAB ${ }^{\mathrm{TM}}$ mesh function, shown in Fig. 2, in which the data points are interpolated by linear functions. In both plots the measured impedance points are indicated with black dots.

The linear function interpolation routine applied in the mesh plot of figure 2 is noticeably discontinuous and requires a large amount of measured data to produce the reasonable surface plot shown here. This plotting routine will also lead to large errors near the edges of the measurement space, which is evident from the jagged nature of the surface edges. Additionally, it is not possible to interpolate the data between the measured points with this mesh plotting routine. The smoothing spline function on the other hand (Fig. 1) is a continuous surface which can easily be evaluated at any point in the impedance space.

The results shown in these figures are measured loadpull data for a Freescale MRF6S19100N power transistor at $1.99 \mathrm{GHz}$. The surface is the CW P-1dB power compression point over the measured load impedance space.

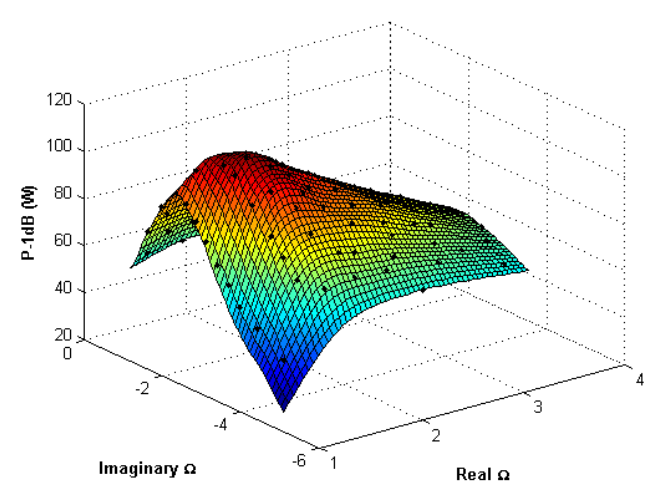

Fig. 1 Thin-plate spline function approximation to measured load pull data of $\mathrm{P}-1 \mathrm{~dB}$ power.

Figs. 3 and 4 show how the thin-plate smoothing spline function can represent exactly the measured load pull data. Fig. 3 shows a simple contour plot of P-1dB power, generated from the measured data, while figure 4 shows a flattened top view of the smoothing spline function. Both plots are identical, which is to be expected because the multivariate spline function will pass through all of the measured data points. Clearly, there is no significant loss in accuracy by representing the complete measured data set using a thin-plate spline approximation function, which leads us to the question of whether the use of thin-plate spline surface modeling techniques will allow for a comparable level of accuracy from a reduced set of measured data?

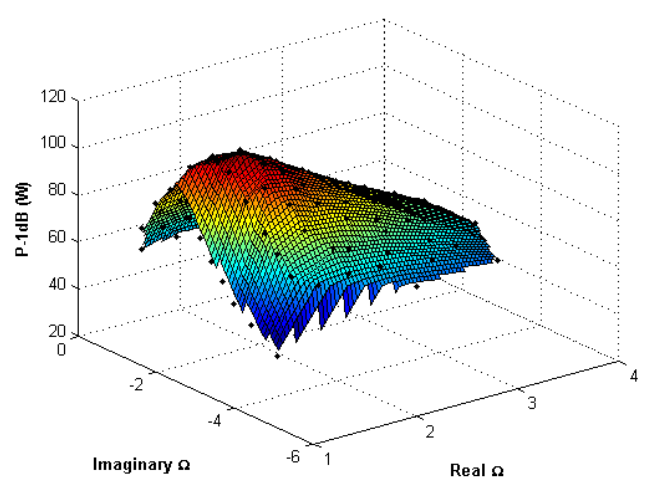

Fig. 2 Simple surface plot of measured P-1dB power using the MATLAB ${ }^{\mathrm{TM}}$ mesh function.

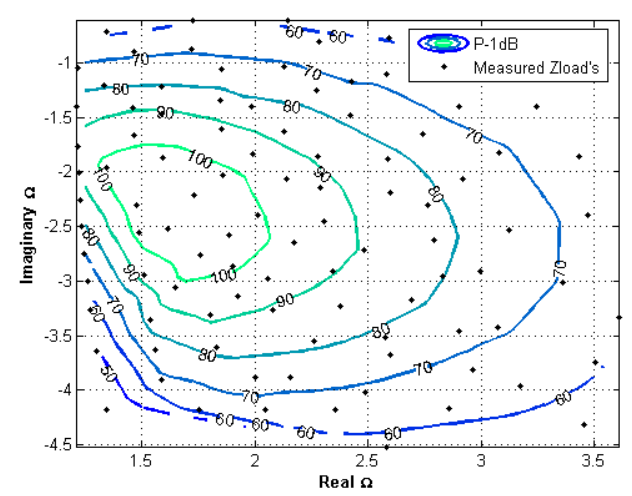

Fig. 3 Simple contour plot of measured P-1dB power.

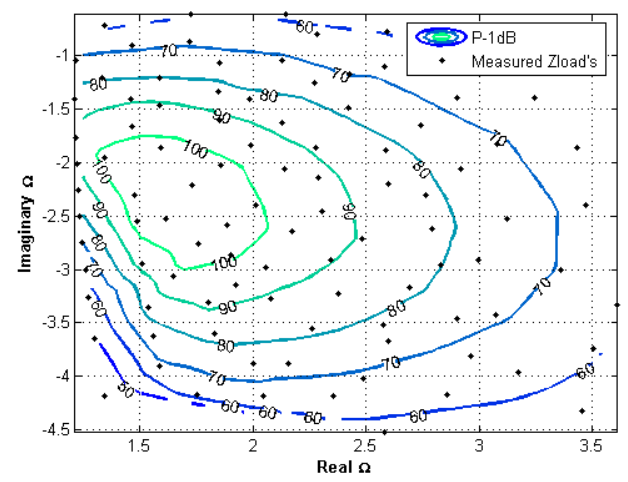

Fig. 4 Top view of the thin-plate spline surface approximation of measured $\mathrm{P}-1 \mathrm{~dB}$ power. 


\section{RESUlTS}

The surface in Fig. 1 and the contours in Fig. 4 are obtained by thin-plate spline interpolation of power measurements taken at over 100 impedance points. In Fig. 5 we show the locations of the measured data points in the impedance plane (black dots). We have selected a subset of 36 points from this data set (blue squares): the selection is currently arbitrary, though we could make the selection based upon the gradient of the contours of P-1dB measurements for example. Using this subset, we have created a new surface using the thin-plate spline function, and in Fig. 6 we plot this new surface, and also the error surface which is calculated as the difference calculated $\mathrm{P}-1 \mathrm{~dB}$ between this surface created from 36 measurements and the original surface (Fig. 1) created from over 100 measurements. As can be seen, the error is very small. The error is almost zero at the peak power point in the surface, which was the region of greatest interest to us during this evaluation, and is greatest at the very edges of the measurement space with a maximum error of approximately $8 \%$. The error is negligible for all of the interior regions of the response surface, which tells us that if the region of greatest interest is enclosed within the measurement space the thin-plate spline approximation is valid.

This has resulted in a reduction in measurement time of over $60 \%$, with no perceptible loss in accuracy, over the same impedance space.

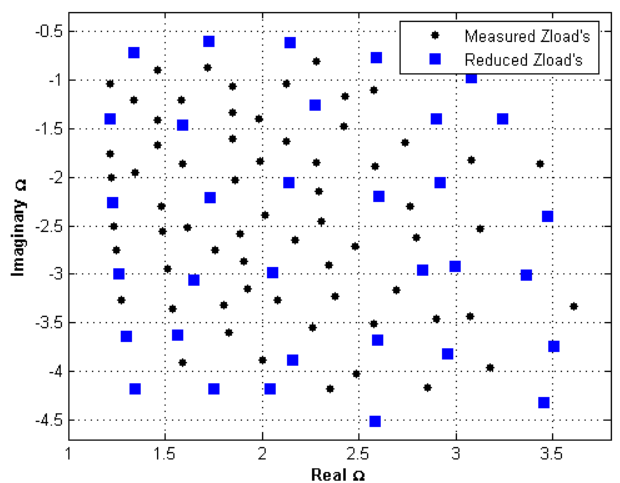

Fig. 5 Measured load impedances - complete and reduced set.

Further, we can make the load-pull measurements over the impedance space over a range of drive powers, and then 'stack' the spline-fitted surfaces, as shown in Fig. 7 for the transistor Gain and Fig. 8 for the transistor PAE. It is then possible to interpolate between the surfaces, at a given, though not necessarily measured, impedance point, to produce the drive-up characteristics for the device at any impedance point we choose. The power of this technique is illustrated in Fig. 10 and Fig. 11 where we show the gain and efficiency of the device respectively as a function of power, interpolated between the response surfaces at a load impedance value that is not in the original measurement data set.
The interpolated Gain and PAE drive-up responses are compared against measured load pull data at the nearest four measured load impedance points to demonstrate the accuracy of interpolating across multiple thin-plate spline surfaces.

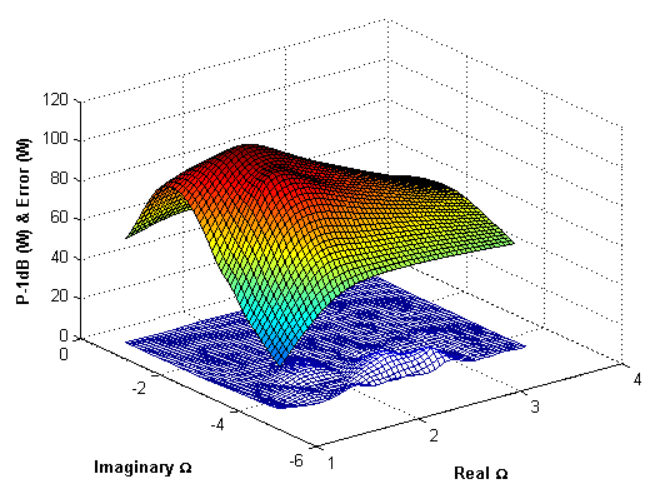

Fig. 6 Thin-plate spline P-1dB surface approximation from reduced data set, and error surface.

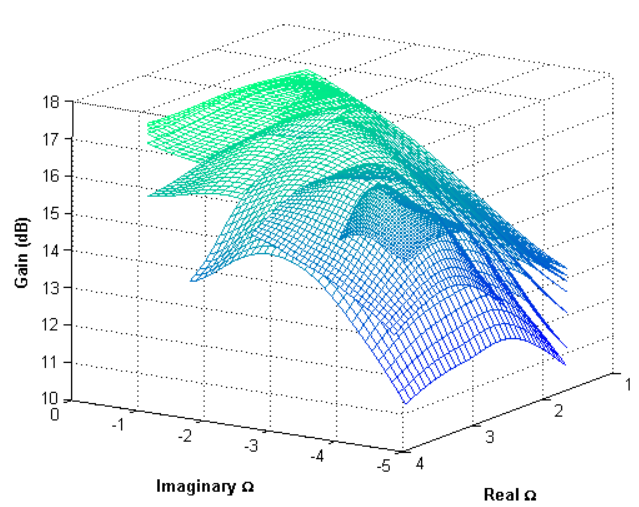

Fig. 7 "Stacked” thin-plate spline approximations of Gain at increasing power levels.

In Fig. 7 the spline surfaces of gain at increasing power levels covering a $15 \mathrm{~dB}$ dynamic range are shown. The smallest (and lowest) gain surface corresponds to the highest power level and the impedance at which this surface is a maximum corresponds to the maximum P-1dB power location.

Fig. 8 shows the corresponding PAE surfaces. Again in this plot, the surface sizes are constant at lower power levels and reduce in size at the highest power levels. The location at which the smallest surface is a maximum corresponds to load impedance at which PAE is maximized at $\mathrm{P}-1 \mathrm{~dB}$

Fig. 9 shows the measured impedance space with the measured and interpolated load impedances which were used to generate the Gain and PAE drive-up plots in Fig. 10 and Fig. 11 indicated. All of the thin-plate spline surfaces of Gain and PAE were generated from the reduced data set (blue squares) of impedances. 


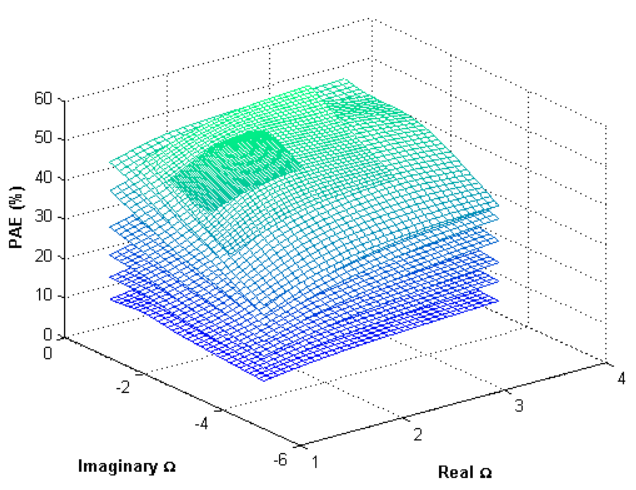

Fig. 8 "Stacked” thin-plate spline approximations of PAE at increasing power levels.

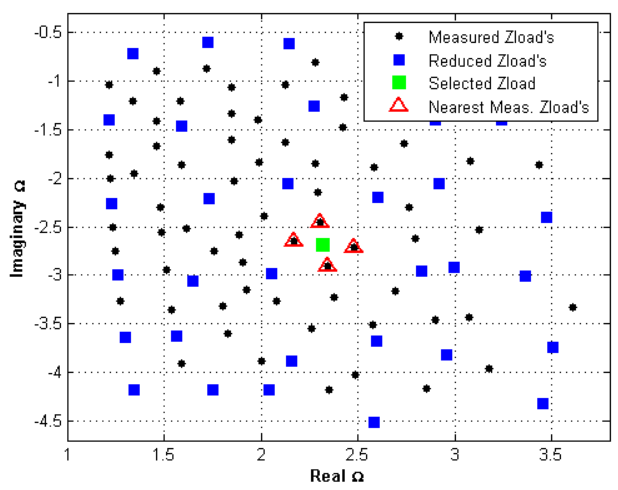

Fig. 9 Measured load impedance space showing measured and interpolated impedances for drive-up plots.

The Gain and PAE drive-up curves shown in Fig. 10 and Fig. 11 are measured load pull data, while the green curves are interpolated from the stacked thin-plate spline surfaces of the reduced data set at the notmeasured load impedance. The interpolated drive-up data falls perfectly between the measured curves illustrating the accuracy of this surface modeling technique.

The interpolated drive-up responses of Gain and PAE were generated from thin-plate response surfaces at discrete, equally spaced power levels, and each response surface was generated from a $60 \%$ reduced data set. It is clear that further reductions in measurement time can be found by reducing the number of power levels at which data is measured, and measuring at non-equally spaced power levels. The number of measured powers can be greatly reduced at backed-off power levels without any additional loss in accuracy.

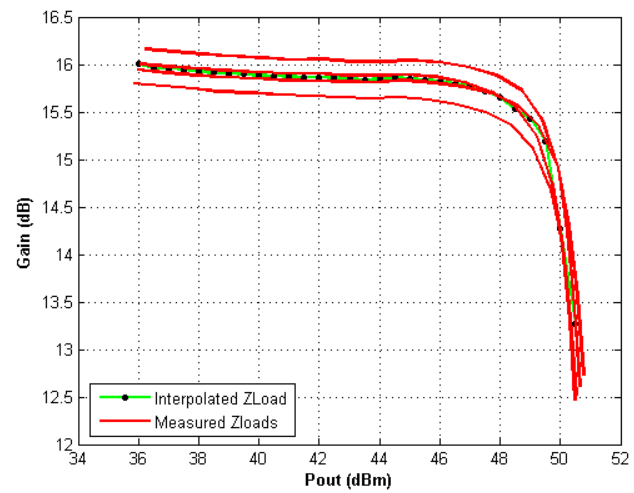

Fig. 10 Drive-up plot of Gain at measured and interpolated load impedances.

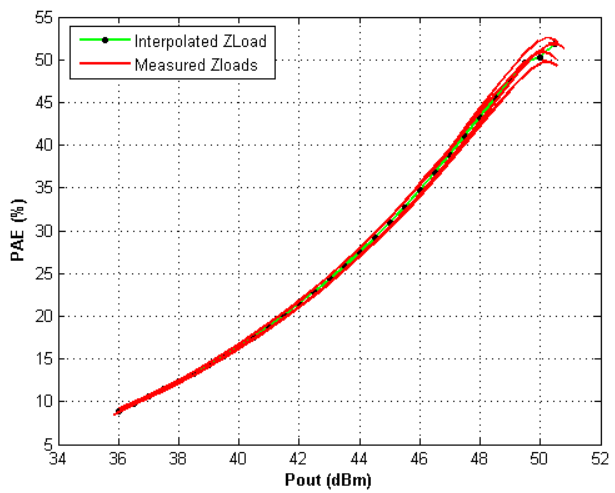

Fig. 11 Drive-up plot of PAE at measured and interpolated load impedances.

\section{CONCLUSIONS}

We have demonstrated that by use of thin-plate splines we can generate an excellent approximation to a measured load-pull surface using a much reduced number of measured impedance points. We have also shown that we can also interpolate between stacked surfaces to obtain accurate drive-up characteristic of the device under test. This has resulted in a significant reduction in measurement time with no loss of accuracy in device characterization.

\section{REFERENCES}

[1] W. Press et al, Numerical Recipes, Cambridge University Press (1990)

[2] S. D. Conte \& C. de Boor, Elementary Numerical Analysis, McGraw-Hill (1972)

[3] Mathworks, Inc., MATLAB ${ }^{\mathrm{TM}}$ Spline Toolbox 3.2.2 User Manual. 